\title{
Prevalence, clinical features, and natural history of incidental clinically non-functioning pituitary adenomas
}

\author{
Pedro Iglesias ${ }^{1}$, Karina Arcano ${ }^{1}$, Vanessa Triviño ${ }^{2}$, Paula García- \\ Sancho ${ }^{3}$, Juan José Díez ${ }^{1,4}$, Carles Villabona ${ }^{3}$, Fernando Cordido ${ }^{2}$ \\ ${ }^{1}$ Department of Endocrinology, Hospital Universitario Ramón y Cajal, Madrid, Spain. \\ ${ }^{2}$ Department of Endocrinology, Hospital Universitario A Coruña, A Coruña, Spain. \\ ${ }^{3}$ Department of Endocrinolgy, Hospital Universitario de Bellvitge, L' Hospitalet de Llobregat, \\ Barcelona, Spain. \\ ${ }^{4}$ Department of Medicine, Universidad de Alcalá de Henares, Madrid, Spain.
}

\begin{abstract}
The objective of the study was to assess the prevalence and clinical features of incidentally discovered clinically non-functioning pituitary adenoma (CNFPA) and to analyze its natural history. A multicenter retrospective study in patients diagnosed with incidental CNFPA periodically followed-up in 3 specialized neuroendocrinology units from 1992 to 2015 was performed. Out of a total of 189 CNFPA patients, 57 cases $(30.1 \%$; 29 women; age at diagnosis $55.8 \pm 16.7$ years) were incidental. Most patients $(n=55,96.5 \%)$ were diagnosed by magnetic resonance imaging (MRI). A sum of $71.9 \%(n=41)$ were macroadenomas; 2 of them $(3.5 \%)$ were giant adenomas $(\geq 4 \mathrm{~cm})$. Patients with macroadenomas were older than those with microadenomas (59.5 \pm 16.7 vs. $46.4 \pm 18.1$ years, $\mathrm{p}=0.007)$. Macroadenomas were more common in men $(85.7 \%$ vs. $58.6 \%$, p=0.023). Twenty-eight patients $(49.1 \%)$ showed suprasellar extension; of these, 19 were accompanied by chiasmatic compression. Hypopituitarism was present in 14 (24.6\%) patients; which was partial in 13 patients $(22.8 \%)$ and complete in one patient $(1.8 \%)$. The gonadal axis was the most frequently affected $(n=13,22.8 \%)$. Twenty four patients $(42.1 \%)$ underwent surgery. Of the non-operated patients, 26 patients could be evaluated morphologically after a median follow-up of 15.5 months (interquartile range, 5.7-32.7 months). No significant changes were found in the maximum tumor diameter at the end of follow-up (1.2 \pm 0.6 vs $1.2 \pm 0.7 \mathrm{~cm})$. The majority of CNFPAs evaluated $(\mathrm{n}=23,88.5 \%)$ did not show any changes in size. In 2 cases $(7.7 \%)$ tumor size decreased, and in one patient $(3.8 \%)$ increased. In our series of CNFPA patients, approximately one-third were incidental. These tumors were diagnosed by MRI preferably from the 5th decade of life without sex predilection. Most of them were macroadenomas, more commonly diagnosed in men and at an older age, compared to microadenomas. The suprasellar extension with chiasmatic compression and hypopituitarism were frequent at diagnosis. Most of the non-operated incidental CNFPAs remain with stable tumor size over time, growth being an unusual event.
\end{abstract}

\section{Key words}

Pituitary incidentaloma, clinically non-functioning pituitary adenoma, restrospective, multicenter study 



\section{Introduction}

Pituitary adenoma (PA) is a neuroendocrine tumor diagnosed with increasing frequency $[1,2]$. At present, it is considered as the third neoplasm of the central nervous system, following to meningioma and glioma [3, 4]. The majority of PAs are functioning, mainly prolactinomas, and approximately one third of the patients have clinically non-functioning pituitary adenomas (CNFPAs) [5, 6].

The increase in the use of imaging techniques in recent years, mainly magnetic resonance imaging (MRI), has been associated with a noteworthy increase in the incidence of pituitary lesions incidentally discovered, also named pituitary incidentalomas (PIs) [7-9]. Its prevalence is very variable and ranges from $11 \%$ in autopsy studies to 4-20\% and 10-38\% in computed tomography (CT) and MRI studies, respectively [10]. More than $90 \%$ of these PIs are pituitary adenomas, usually CNFPAs, and about $9 \%$ are craniopharyngiomas or Rathke's cleft cysts $[9,11,12]$.

CNFPAs in adults are usually macroadenomas $(\geq 1 \mathrm{~cm})$ and frequently are associated with compressive symptoms derived from local involvement of neighboring structures with neuro-ophthalmological symptoms and/or hypopituitarism. However, macro-CN-FPAs can be also incidental tumors discovered in imaging study. On the contrary, micro-CNFPAs $(<1 \mathrm{~cm})$ are almost always incidentally discovered lesions.

Although the prevalence of PIs has significantly increased in re-cent years, data regarding the prevalence and natural history of CNFPAs incidentally discovered remain sparse $[6,7,10,13-15]$. Therefore, our aims were 1) to know the prevalence of incidental CNFPAs from a cohort of CNFPA patients, 2) to assess the clinical features of these incidental CNFPAs, and 3) to analyze its natural history in non-operated patients.

\section{Patients and Methods}

\section{Patients}

A multicenter retrospective study in patients diagnosed with incidental CNFPA and periodically followed-up in 3 specialized Neuroendocrinology Units from 3 different tertiary care Spanish hospitals in the last 4 decades (from 1992 to 2015) was performed.

Patient distribution according to location was Hospital Ramón y Cajal, Madrid, $\mathrm{n}=$ 27 (47.4 \%); Hospital Universitario A Coruña, A Coruña, $\mathrm{n}=18$ (31\%); and Hospital de Bellvitge, L'Hospitalet de Llobregat, Barcelona, $\mathrm{n}=12$ (21.1\%). Median (interquartile range) follow-up was 25 months (7-127 months).

\section{Methods}

PI was considered when a previously unsuspected pituitary lesion was found in a casual way after performing an imaging study for reasons unrelated to pituitary disease. Incidental CNFPA was de-fined as a PI compatible with PA in imaging study in the absence of clinical evidence of pituitary hormonal overproduction in non-operated patients or the histological demonstration of PA in those patients underwent surgery. All those patients with imaging study compatible with craniopharyngioma and Rahtke's cleft cyst were excluded. We also excluded non-operated patients treated with 
radiotherapy and/or medical treatment (dopaminergic agonists and/or somatostatin analogues). 
For every patient, clinical parameters (age at diagnosis, sex, and main complaint at presentation), endocrine dysfunction (the presence of hypopituitarism, number of axes involved, and hyperprolactinemia), ophthalmological symptoms, imaging study (MRI and/or CT scan findings), and change in tumor size were recorded.

The surgical criteria were individualized for each patient according to medical criterion, clinical situation, and acceptance of surgery by the patient. These criteria were based primarily on the mass effect of the tumor associated with neuro-ophthalmological involvement with or without pituitary hypofunction.

All procedures performed in the present study were in accordance with the ethical standards of the institutional committee and with the 1964 Helsinki declaration.

\section{Statistical analysis}

For quantitative variables, results are expressed as mean \pm SD for normally distributed data, and as median (interquartile range) for nonparametric data. Adjustment to normal distribution was tested by the Kolmogorov test. Categorical variables are described as percentages. For comparisons of means between 2 groups of sub-jects the Student's t-test was used for normally distributed data, and the Mann-Whitney test was employed for nonparametric data. For categorical comparisons the $\chi^{2}$ test or the Fisher exact test were used. Differences were considered significant when $\mathrm{p}<0.05$.

\section{Results}

\section{Clinical and hormonal data}

Of a total of 189 patients with CNFPA, 57 cases (30.1\%) [29 women (50.9\%); age at diagnosis $55.8 \pm 16.7$ years (range, 18-83)] were incidental. Males were older than females $(59.3 \pm 14.6$ vs. $52.4 \pm 18.2$ years, ns). Of incidental CNFPAs, $43.9 \%$ were older than 60 years, $40.3 \%$ belonged to the age group of 40-60 years and $15.8 \%$ were younger than 40 years. Clinical features of the patients according to clinical form presentation at diagnosis are summarized in Table 1. Distribution of 57 patients with incidental CNF-PAs according to sex and adenoma size at diagnosis is shown in $\bullet$ Fig. 1 . 
-Table 1. Clinical features of 189 patients with clinically non-functioning pituitary adenomas according to clinical form presentation at diagnosis.

\begin{tabular}{|c|c|c|}
\hline & Incidental $\mathrm{n}=57(30.1 \%)$ & Non-incidental $\mathrm{n}=132(69.9 \%)$ \\
\hline \multicolumn{3}{|l|}{ Clinical data at diagnosis } \\
\hline Age $($ mean \pm SD) $($ years $)$ & $55.8 \pm 16.7$ & $51.5 \pm 15.8$ \\
\hline $\operatorname{Sex}(M / F), n(\%)$ & $28(49.1 \%) / 29(50.9 \%)$ & $62(47 \%) / 70(53 \%)$ \\
\hline \multicolumn{3}{|l|}{ Age groups, n (\%) } \\
\hline$<40$ years & $9(15.8 \%)$ & $27(20.4 \%)$ \\
\hline $40-60$ years & $23(40.3 \%)$ & $69(52.3 \%)$ \\
\hline$>60$ years & $25(43.9)$ & $36(27.3 \%)$ \\
\hline Abnormal visual field, n (\%) & $18(31.5 \%)$ & $60(45.4 \%) *$ \\
\hline \multicolumn{3}{|l|}{ Endocrinological study, n (\%) } \\
\hline Hypopituitarism & $14(24.6 \%)$ & $49(37.1 \%) *$ \\
\hline Partial & $13(22.8 \%)$ & $41.8(32 \%) *$ \\
\hline Complete & $1(1.8 \%)$ & $7(5.3 \%)$ \\
\hline Hyperprolactinemia & $14(24.6 \%)$ & $32(24.2 \%)$ \\
\hline Central hypogonadism & $13(22.8 \%)$ & $37(28.0 \%)$ \\
\hline Central hypothyroidism & $9(15.8 \%)$ & $24(18.2 \%)$ \\
\hline Secondary adrenal insufficiency & $7(12.3 \%)$ & $26(19.7 \%)$ \\
\hline GH deficiency & $3(5.3 \%)$ & $18(13.6 \%)$ \\
\hline \multicolumn{3}{|l|}{ Imaging study, n (\%) } \\
\hline MRI & $55(96.5 \%)$ & $120(90.9 \%)$ \\
\hline $\mathrm{CT}$ & $2(3.5 \%)$ & $12(9.1 \%)$ \\
\hline Suprasellar extension & $28(49.1 \%)$ & $92(69.7 \%) * *$ \\
\hline Chiasma compression & $19(33.3 \%)$ & $79(59.8 \%) * * *$ \\
\hline Cavernous sinuses invasion & $16(28.1 \%)$ & $48(36.4 \%)$ \\
\hline \multicolumn{3}{|l|}{ Tumor related data } \\
\hline Macroadenoma, n (\%) & $41(71.9 \%)$ & $116(87.9 \%) *$ \\
\hline Giant adenoma, n (\%) & $2(3.5 \%)$ & $17(12.9 \%) *$ \\
\hline Microadenoma, n (\%) & $16(28.1 \%)$ & $16(12.1 \%) *$ \\
\hline Maximum tumor diameter $(\mathrm{cm})$ & $1.6 \pm 0.9$ & $2.5 \pm 1.3 *$ \\
\hline
\end{tabular}

$* \mathrm{p}<0.05 ; * * \mathrm{p}<0.01 ; * * * \mathrm{p}<0.001$ non-incidental vs. incidental CNFPA.

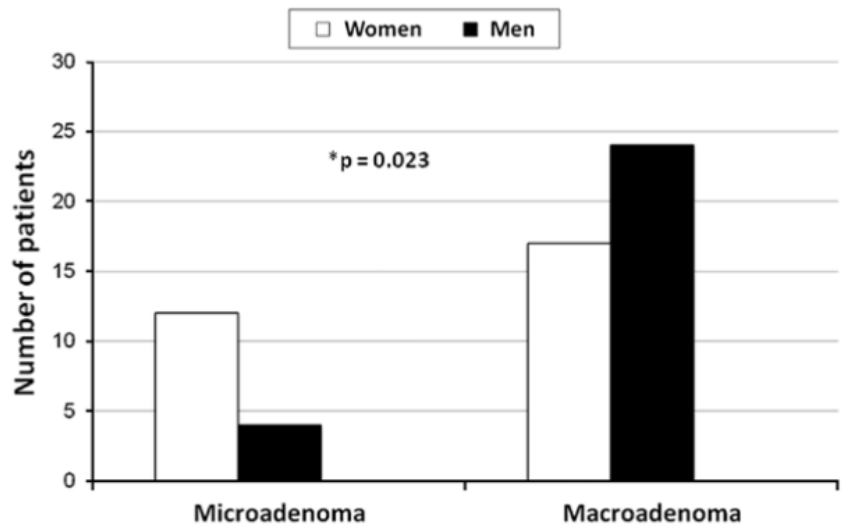

-Fig. 1. Distribution of 57 patients with incidental clinically non-functioning pituitary adenomas according to sex and adenoma size at diagnosis. 
Most patients $(\mathrm{n}=55,96.5 \%)$ were diagnosed by MRI and the remaining by CT. Hyperprolactinemia was present in 14 patients $(24.6 \%)$. The median prolactin level was $11.1 \mathrm{ng} / \mathrm{ml}(7-21.1 \mathrm{ng} / \mathrm{ml}$; range 2.6-95 ng/ml). Hyperprolactinemic patients showed a higher tumor size than normoprolactinemic patients $(2.3 \pm 0.9 \mathrm{~cm}$ vs. $1.4 \pm 0.9 \mathrm{~cm}, \mathrm{p}=$ 0.006). The percentage of patients underwent surgery was significantly higher in hyperprolactinemic than in normoprolactinemic patients $(73.3 \%$ vs. $28.5 \%, \mathrm{p}=0.002)$. No significant difference in the prevalence of hyperprolactinemia between inci-dental and non-incidental CNFPAs was found ( $\bullet$ Table 1$)$.

Hypopituitarism was present in $14(24.6 \%)$ patients; which was partial in 13 patients $(22.8 \%)$ and complete in one patient $(1.8 \%)$. The gonadal axis was the most frequently affected $(n=13,22.8 \%)$. Incidental CNFPA patients who underwent surgery showed a higher prevalence of hormonal involvement of the gonadal $(\mathrm{p}=0.008)$, adrenal $(\mathrm{p}=0.034)$, and thyroid $(\mathrm{p}=0.028)$ axes compared to those not operated. Lastly, incidental CNFPAs were associated with less involvement of pituitary function than those with non-incidental CNFPAs ( Table 1).

\section{Tumor-related data}

Forty one patients $(71.9 \%)$ had macroadenomas; 2 of them $(3.5 \%)$ were giant adenomas $(\geq 4 \mathrm{~cm})$. Patients with macroadenomas were older than those with microadenomas $(59.5 \pm 16.7$ vs. $46.4 \pm 18.1$ years, $\mathrm{p}=0.007)$. Macroadenomas were more common in men $(85.7 \%$ vs. $58.6 \%, \mathrm{p}=0.023)(\vee$ Fig. 1$)$.Mean maximum tumor diameter was $1.6 \pm 0.9 \mathrm{~cm}$ and was significantly higher in males than in females $(2,0 \pm$ 1.0 vs. $1.3 \pm 0.8 \mathrm{~cm} ; \mathrm{p}=0.015$ ) ( $\bullet$ Fig. 2 ). Incidental CNFPAs were significantly smaller in size and less invasive than non-incidental CNFPAs ( Table 1).

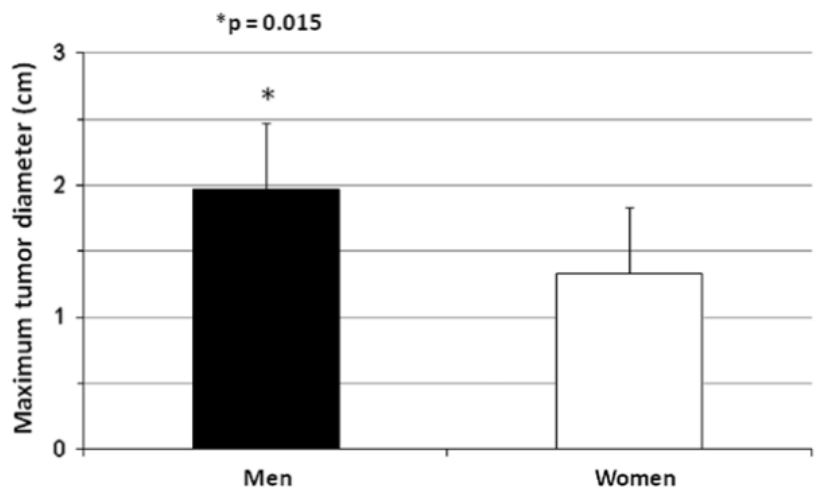

-Fig. 2. Maximum tumor diameter at diagnosis according to sex in 57 patients with incidental clinically non-functioning pituitary adenomas.

About half of the patients $(n=28 ; 49.1 \%)$ showed suprasellar extension; of these, 19 (33.3\%) were accompanied by chiasmatic compression. Abnormal visual field examination was common $(\mathrm{n}=18,31.5 \%)$ although its prevalence was lower than that 
observed in non-incidental CNFPAs (45.4\%, p $=0.028)$. Invasion of the cavernous sinuses was present in $28.1 \%(\mathrm{n}=16)$. 
Twenty four patients (42.1\%) underwent surgery. Hypopituitarism $(\mathrm{p}=0.002)$, central hypogonadism $(\mathrm{p}=0.004)$, secondary adrenal insufficiency $(\mathrm{p}=0.013)$, central hypothyroidism $(\mathrm{p}=0.019)$, and hyperprolactinemia $(\mathrm{p}=0.002)$ were more common in these patients than in those not surgically treated. Immunohistochemical study for anterior pituitary hormones was performed in the tumor samples of the patients. Hormones most frequently detected were gonadotropins ( $\mathrm{FSH}[\mathrm{n}=5,20.8 \%]$ and $\mathrm{LH}[\mathrm{n}$ $=4,16.7 \%])$, followed by PRL $(n=2,8.3 \%)$, ACTH $(n=1,4.2 \%)$, and GH $(n=1,4.2$ $\%)$. None of the patient showed TSH positive staining. In 9 patients $(37.5 \%)$, immunohistochemical staining was negative.

Of the 33 non-operated patients, 26 patients $(45.6 \%)$ could be evaluated after a median follow-up of 15.5 months (interquartile range, 5.7-32.7 months). No significant changes were found in the maximum tumor diameter at the end of follow-up $(1.2 \pm 0.6$ vs. $1.2 \pm 0.7 \mathrm{~cm}$; NS). The majority of CNFPAs evaluated $(\mathrm{n}=23,88.5 \%)$ did not show any changes in size. In 2 cases $(7.7 \%)$, tumor size de-creased (reduction of maximum diameter $>2 \mathrm{~mm}$ ) and in one patient $(3.8 \%)$ increased (increase of maximal diameter $>2 \mathrm{~mm}$; initial maximal tumor diameter $1.0 \mathrm{~cm} ; 1.3 \mathrm{~cm}$ increase in a 30-yearold male after 76 months of follow-up) ( $\bullet$ Fig. 3).

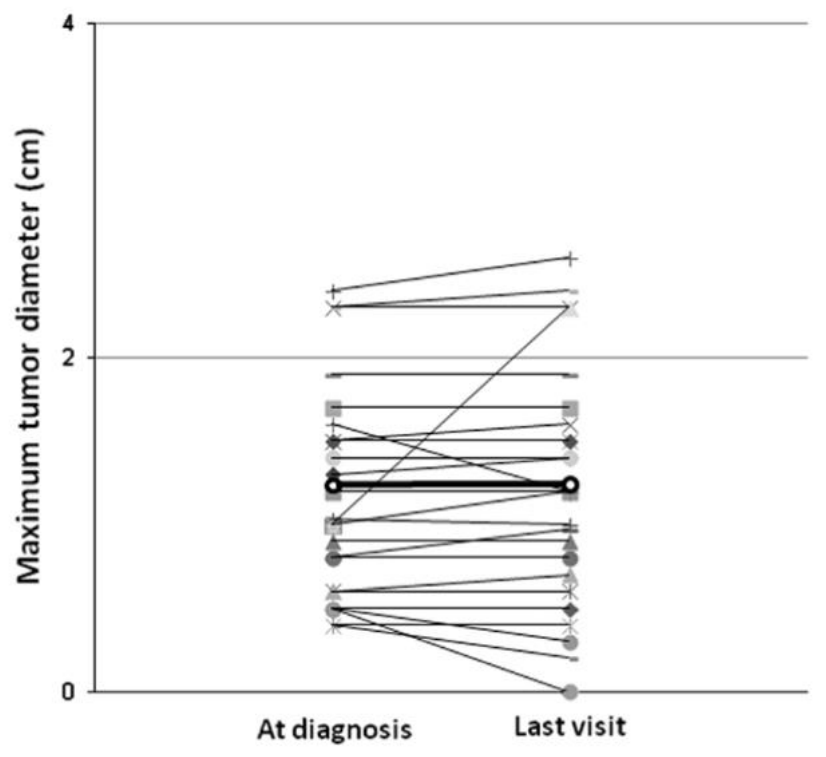

-Fig. 3. Spontaneous evolution of pituitary adenoma size in 26 patients with incidental clinically non-functioning pituitary adenomas after a median follow-up of 15.5 months (interquartile range, 5.7-32.7 months). The black thick line joins the points representing the mean maximum tumor diameter at diagnosis and at the last visit. 


\section{Discussion}

The present study shows that in our environment CNFPAs were incidentally discovered in about one third of cases. In spite of being tumors discovered incidentally, a detailed clinical, analytical, ophthalmologic and morphological study reveals that approximately one third shows chiasmatic compression and about a quarter is associated with pituitary hypofunction, although hypopituitarism is usually partial. These incidental CNFPAs are usually macroadenomas (approximately 3 quarters) commonly discovered at around 60 years of age. The prevalence of tumor growth after a roughly 1 year of follow up in non-operated incidental CNFPAs is very low $(\sim 4 \%)$.

Most studies that have analyzed PIs have considered all types of pituitary lesions incidentally found in the imaging study [13]. In this setting, they are not homogeneous series of patients and, therefore, the results are not applicable to a particular pathology. One of the main differences between our study and the rest of the studies carried out on PIs is that we excluded all those patients with other pituitary lesions such as craniopharyngiomas, pituitary cysts, and functioning pituitary adenomas, therefore only patients with a clinical diagnosis of CNFPA were considered.

It has been reported that about half (45\%) of PIs are macroincidentalomas [10]. In our series of 189 CNFPA patients, approximately one third $(n=57)$ were incidentally found. Most of these patients $(n=41,71.9 \%)$ had macroadenomas. This prevalence, although elevated, was significantly lower than that observed in non-incidental CNFPAs $(87.9 \%)$.

Mean age at diagnosis of PIs is around 47 years, with a slightly higher age in males compared to females [16]. In our cohort of incidental CNFPA patients age at diagnosis was higher not only to that reported in PIs, but also to the non-incidental CNFPAs, although in the latter case statistical significance was not reached (56 years vs. 51 years, respectively). It is possible that the different etiologies of PIs and the presence of symptoms associated with non-incidental CNFPAs would explain these differences. As reported in PIs [16, 17], macroadenomas were more frequently found in males with incidental CNFPAs. The contrary occurred with microadenomas, which were more commonly seen in women. It is possible that CNFPAs in men have a higher proliferative activity and tumor aggressiveness showing a different nature and biological behavior compared to women, as it has been suggested in other pituitary tumors such as prolactinomas [18].

MRI is considered as the gold standard for initial imaging diagnosis of sellar lesions providing more detailed images relative to CT. In our study, the great majority of incidental CNFPAs were discovered by this technique. Less than $5 \%$ of these tumors were dis-covered by CT preferably in the hospital emergency services where CT is the preferred imaging technique. Some preoperative MRI appearances of pituitary adenomas have been associated with CNFPA such as lowered T1 relaxation rate and increased tumor extension into adjacent anatomic compartments and sinus invasion [19].

Hyperprolactinemia is a common finding in CNFPAs. Its prevalence in patients with histologically proven non-functioning adenomas is 25-65\% [20]. In a retrospective study of 226 CNFPA patients, median prolactin level was $18 \mathrm{ng} / \mathrm{ml}$ (range $0.8-73.6$ $\mathrm{ng} / \mathrm{ml}$ ) [21]. Although the differentiation between CNFPA with hyperprolactinemia and prolactinoma is sometimes difficult, it has been suggested that prolactin levels < 100 $\mathrm{ng} / \mathrm{ml}$ associated with older age, extrasellar extension with visual defects and the presence of hypo-functioning GH axis can be considered suggestive of CNFPA [22]. Our incidental CNFPA patients showed similar prolactin levels $(11.1 \mathrm{ng} / \mathrm{ml})$ and similar 
prevalence $(24.6 \%)$ of hyperprolactinemia than those reported by others in nonincidental CNFPA patients [20], suggesting that hyperprolactinemia is a common finding in patients with CNFAs regardless of clinical presentation, that is, incidental or symptomatic, where significant differences in the prevalence of hyperprolactinemia were not found. 
Multiple observational and retrospective studies performed in CNFPA patients have shown a prevalence of partial and complete hypopituitarism of 37-85\% and 6-29\%, respectively [20]. In our series these percentages were similar or slightly lower for nonincidental CNFPAs (32\% and $5.3 \%$, respectively). In relation to incidental CNFPAs, we found a significantly lower prevalence of partial hypopituitarism compared with non-incidental CPNAs. In spite of this, this prevalence was high $(22.8 \%)$ indicating that, in many cases, the symptoms associated with hormonal deficiency are un-noticed or non-referred by the patient. GH deficiency and central hypogonadism have been reported to be the most commonly affected axes in CNFPAs, followed by corticotropin and thyrotropin deficiencies [20]. In our series of incidental CNFPAs, gonadotropin deficiency was the endocrine pituitary hypofunction more commonly reported, followed by thyrotropin and corticotropin deficiencies, and finally GH deficiency. These results were very similar to those found in non-incidental CNFPAs, indicating that there does not seem to be a relationship between the type of hormonal pituitary deficiency and the clinical presentation form (symptomatic or incidental) of CNFPAs.

Based on the available clinical evidence, mainly retrospective and prospective observational cohort studies, pituitary surgery is the treatment of choice in symptomatic CNFPA patients and in those who show tumor growth during follow-up. Surgery is accompanied by a significant reduction in tumor size, and a significant improvement in both visual and pituitary functions [23]. It is note-worthy that in our series of incidental CNFPAs, a high percentage $(42.1 \%)$ was treated with surgery suggesting a high prevalence of non-perceived symptoms (neuroophtalmologic and/or endocrine symptoms) in these patients. Lastly, a significant higher prevalence of pituitary hypofunction in incidental CNFPAs underwent surgery compared with not-surgically treated patients was found.

Few studies have evaluated the natural history of CNFPAs. An analysis of the natural history of 513 CNFPAs in 10 series of patients during a 2 to 7 -year follow-up showed that most of them did not modify tumor size $(83.1 \%$ and $63.2 \%$ for micro- and macroadenomas, respectively). An increase in tumor size was reported in $10.6 \%$ of the microadenomas and $24.1 \%$ of the macroadenomas, while a spontaneous reduction of the tumor volume was reported in $6.3 \%$ and $12.7 \%$ of the micro- and macroadenomas, respectively [14]. However, not all patients in this series were incidental; many of them did not undergo surgery due to major comorbidities or refusal of surgery by the patient. To the best of our knowledge, our series is the first to analyze only patients with incidental CNFPAs with-out surgical criteria. In this case the majority of tumors remained stable $(88.5 \%)$, a minority decreased $(7.7 \%)$, and tumor growth was exceptional $(3.8 \%)$. These findings suggest that clinical follow-up with endocrine evaluation and imaging studies is a reason-able option in these patients.

In conclusion, CNFPAs are diagnosed incidentally in about one third of cases, usually by MRI preferably from the 5th decade of life without sex predilection. Most of them are macroadenomas, more commonly diagnosed in men and at an older age, compared to microadenomas. Suprasellar extension with chiasmatic compression and visual field defects and hypopituitarism are frequent at diagnosis. Most of the nonoperated incidental CNFPAs remain with stable tumor size over time, growth being an unusual event.

\section{Funding}

The work was supported in part by FIS del Instituto de Salud Carlos III PI16/00884 (FEDER from E.U.), Spain. 


\section{Conflicts of interest}

The authors declare that they have no conflict of interest. 


\section{References}

[1] Ezzat S, Asa SL, Couldwell WT, Barr CE, Dodge WE, Vance ML, McCutcheon IE. The prevalence of pituitary adenomas: A systematic review. Cancer 2004; 101: 613-619

[2] Aflorei ED, Korbonits M. Epidemiology and etiopathogenesis of pituitary adenomas. J Neurooncol 2014; 117: 379-394

[3] Dolecek TA, Propp JM, Stroup NE, Kruchko C. CBTRUS statistical report: primary brain and central nervous system tumors diagnosed in the United States in 2005-2009. Neuro Oncol 2012; 14 (Suppl. 05) v1-49

[4] Shibui S. The present status and trend of brain tumors based on the data of the Brain Tumor Registry of Japan. Brain Nerve 2012; 64: 286-290

[5] Mindermann T, Wilson CB. Age-related and gender-related occurrence of pituitary adenomas. Clin Endocrinol (Oxf) 1994; 41: 359-364

[6] Molitch ME. Diagnosis and treatment of pituitary adenomas: A review. JAMA 2017; 317: 516-524

[7] Molitch ME. Nonfunctioning pituitary tumors. Handb Clin Neurol 2014; 124: 167-184

[8] Scangas GA, Laws Jr. ER. Pituitary incidentalomas. Pituitary 2014; 17: 486-491

[9] Vasilev V, Rostomyan L, Daly AF, Potorac I, Zacharieva S, Bonneville JF, Beckers A. Management of endocrine disease: Pituitary 'incidentaloma': neuroradiological assessment and differential diagnosis. Eur J Endocrinol 2016; 175: R171-R184

[10] Freda PU, Beckers AM, Katznelson L, Molitch ME, Montori VM, Post KD, Vance ML. Endocrine Society. Pituitary incidentaloma: An endocrine society clinical practice guideline. J Clin Endocrinol Metab 2011; 96: 894-904

[11] Freda PU, Post KD. Differential diagnosis of sellar masses. Endocrinol Metab Clin North Am 1999; 28: 81-117 vi

[12] Esteves C, Neves C, Augusto L, Menezes J, Pereira J, Bernardes I, Fonseca J, Carvalho D. Pituitary incidentalomas: Analysis of a neuroradiological cohort. Pituitary 2015; 18: 777-781

[13] Sivakumar W, Chamoun R, Nguyen V, Couldwell WT. Incidental pituitary adenomas. Neurosurg Focus 2011; 31: E18

[14] Molitch ME. Management of incidentally found nonfunctional pituitary tumors. Neurosurg Clin N Am 2012; 23: 543-553

[15] Bancos I, Natt N, Murad MH, Montori VM. Evidence-based endocrinology: Illustrating its principles in the management of patients with pituitary incidentalomas. Best Pract Res Clin Endocrinol Metab 2012; 26: 9-19

[16] Feldkamp J, Santen R, Harms E, Aulich A, Modder U, Scherbaum WA. Incidentally discovered pituitary lesions: High frequency of macroadenomas and hormone-secreting adenomas - results of a prospective study. Clin Endocrinol (Oxf) 1999; 51: 109-113

[17] Fainstein Day P, Guitelman M, Artese R, Fiszledjer L, Chervin A, Vitale NM, Stalldecker G, De Miguel V, Cornalo D, Alfieri A, Mallea Gil MS. Retrospective multicentric study of pituitary incidentalomas. Pituitary 2004; 7: 145-148

[18] Iglesias P, Bernal C, Villabona C, Castro JC, Arrieta F, Diez JJ. Prolactinomas in men: A multicentre and retrospective analysis of treatment outcome. Clin Endocrinol (Oxf) 2012; 77: 281-287

[19] Chen CC, Carter BS, Wang R, Patel KS, Hess C, Bodach ME, Tumialan LM, Oyesiku NM, Patil CG, Litvack Z, Zada G, Aghi MK. Congress of neurological surgeons systematic review and evidence-based guideline on preoperative imaging assessment of patients with suspected nonfunctioning pituitary adenomas. Neurosurgery 2016; 79: E524-E526

[20] Fleseriu M, Bodach ME, Tumialan LM, Bonert V, Oyesiku NM, Patil CG, Litvack Z, Aghi MK, Zada G. Congress of neurological surgeons systematic review and evidence-based guideline for pretreatment endocrine evaluation of patients with nonfunctioning pituitary adenomas. Neurosurgery 2016; 79: E527-E529

[21] Karavitaki N, Thanabalasingham G, Shore HC, Trifanescu R, Ansorge O, Meston N, Turner HE, Wass JA. Do the limits of serum prolactin in disconnection hyperprolactinaemia need redefinition? A study of 226 patients with histologically verified non-functioning pituitary macroadenoma. Clin Endocrinol (Oxf) 2006; 65: 524-529

[22] Hong JW, Lee MK, Kim SH, Lee EJ. Discrimination of prolactinoma from hyperprolactinemic non-functioning adenoma. Endocrine 2010; 37: 140-147

[23] Lucas JW, Bodach ME, Tumialan LM, Oyesiku NM, Patil CG, Litvack Z, Aghi MK, Zada G. Congress of neurological surgeons systematic review and evidence-based guideline on primary management of patients with nonfunctioning pituitary adenomas. Neurosurgery 2016; 79: E533-E535 